\title{
Aspirina en la prevención primaria. Metaanálisis estratificado por riesgo cardiovascular basal
}

\author{
Aspirin in primary prevention. Meta-analysis stratified by baseline cardiovascular risk
}

Gerardo Masson ${ }^{1,2 *}$, Martín Lobo², Walter Masson ${ }^{2}$ y Graciela Molinero²

${ }^{1}$ Servicio de Cardiología, Instituto Cardiovascular San Isidro; ${ }^{2}$ Consejo de Prevención y Epidemiología, Sociedad Argentina de Cardiología. Buenos Aires, Argentina

\begin{abstract}
Resumen
Introducción: La utilidad de la aspirina en la prevención primaria es todavía objeto de controversia. Los avances médicos y la variabilidad del riesgo cardiovascular podrían explicar la heterogeneidad de los estudios publicados, y las poblaciones de alto riesgo tendrían mayor beneficio. Objetivo: Analizar los efectos de la aspirina en pacientes sin antecedentes cardiovasculares y evaluar los resultados de acuerdo con el riesgo cardiovascular de las poblaciones. Métodos: Se incluyeron estudios que evaluaron el uso de la aspirina en comparación con placebo en la prevención primaria. Se analizó la combinación de muerte cardiovascular, infarto agudo de miocardio (IAM) y accidente cerebrovascular (ACV) isquémico. El punto final de seguridad fue la combinación de ACV hemorrágico y sangrado mayor. Se clasificaron los estudios en riesgo bajo y moderado/alto, de acuerdo con el número de episodios en la rama de placebo. Resultados: Se evaluaron 13 estudios ( $n=164,225)$, ocho de riesgo cardiovascular bajo $(n=118,455)$ y cinco de moderado/alto $(n=45,770)$. Se observó una reducción del punto final combinado en el grupo de aspirina (OR 0.90; IC 95\%, 0.85-0.94), sin diferencias en mortalidad cardiovascular (OR 0.94; IC 95\%, 0.86-1.04). No se identificaron diferencias entre los subgrupos de riesgo. Se reconocieron mayores complicaciones hemorrágicas en el grupo de aspirina (OR 1.45; IC 95\%, 1.32-1.60), sin diferencias entre los subgrupos de riesgo. Conclusión: La aspirina se relacionó con una leve disminución de IAM y ACV isquémico en términos absolutos, sin diferencias en la mortalidad cardiovascular. Esto, junto con el aumento de las complicaciones hemorrágicas, se traduce en una ausencia de beneficio clínico neto. El riesgo cardiovascular basal de la población no modificó los resultados.
\end{abstract}

Palabras clave: Prevención cardiovascular. Prevención primaria. Aspirina.

\begin{abstract}
Background: The usefulness of aspirin in primary prevention continues to be the subject of debate. Medical advances and the variability of cardiovascular risk could explain the heterogeneity of the published studies. High risk populations would have greater benefit. Objective: Analyzing the effects of aspirin in patients without cardiovascular disease and evaluating the results according to the cardiovascular risk of the populations. Methods: Studies evaluating aspirin versus placebo in primary pre-
\end{abstract}

\section{Correspondencia:}

*Gerardo Masson

E-mail: gmasson84@gmail.com
Disponible en internet: 09-03-2020 Arch Cardiol Mex. 2020;90(3):293-299 www.archivoscardiologia.com 1405-9940/๑ 2020 Instituto Nacional de Cardiología Ignacio Chávez. Publicado por Permanyer. Este es un artículo open access bajo la licencia CC BY-NC-ND (http://creativecommons.org/licenses/by-nc-nd/4.0/). 
vention were included. The primary endpoint was the combined cardiovascular death, acute myocardial infarction (AMI) and ischemic stroke. The final safety point was the combination of hemorrhagic stroke and major bleeding. The studies were classified into low and moderate/high risk, according to the number of events in the placebo arm. Results: Thirteen studies were evaluated $(n=164,225)$, eight of low cardiovascular risk $(n=118,455)$ and five of moderate/high risk $(n=45,770)$. There was a reduction of the combined endpoint in the aspirin group (odds ratio [OR] 0.90; 95\% confidence interval [CI], 0.85-0.94), without differences in cardiovascular mortality (OR 0.94; $95 \% \mathrm{Cl}, 0.86-1.04)$. No differences were observed when comparing the risk subgroups. Greater hemorrhagic complications were observed in the aspirin group (OR 1.45; 95\% Cl, 1.32-1.60), without differences between the risk subgroups. Conclusion: Aspirin was associated with a slight decrease in $A M I$ and ischemic stroke in absolute terms, with no differences in cardiovascular mortality. This accompanied by the increase in hemorrhagic complications, results in an absence of net clinical benefit. The baseline cardiovascular risk of the population did not affect the results.

Key words: Cardiovascular prevention. Primary prevention. Aspirin.

\section{Introducción}

Muchos años han pasado y la utilidad de la aspirina en la prevención primaria es todavía un interrogante difícil de responder. Múltiples estudios y diversos metaanálisis han investigado durante las últimas tres décadas el efecto protector de este fármaco en pacientes sin antecedentes cardiovasculares. Sin embargo, aún es en la actualidad un tema de controversia y las distintas sociedades científicas no publican indicaciones precisas en este escenario ${ }^{1,2}$.

El principal motivo de este punto es la heterogeneidad de los resultados de los distintos estudios, sobre todo al analizar los primeros trabajos publicados a fines del siglo pasado y principios del corriente. Las hipótesis que han surgido para explicar estas diferencias son múltiples, pero destaca el avance en el tratamiento de los factores de riesgo cardiovascular y la variabilidad del riesgo cardiovascular de las poblaciones incluidas ${ }^{3}$. En este sentido, un mayor riesgo se acompaña de un mayor número de episodios y, en consecuencia, los beneficios del tratamiento antiagregante en términos absolutos son mayores, cerca de lo observado en la prevención secundaria. Por esta razón, diversos autores han intentado seleccionar mediante la utilización de distintas variables clínicas a subpoblaciones de alto riesgo. Sin embargo, este riesgo está sobreestimado en muchos estudios y el número real de efectos observados es bajo. En otras palabras, el "riesgo cardiovascular real" de estos pacientes fue menor al esperado.

En fecha reciente, tres grandes estudios clínicos han evaluado el consumo de aspirina en diferentes subpoblaciones y han reavivado el debate ${ }^{4-6}$. Asimismo, se ha publicado un metaanálisis, que si bien incluye $y$ analiza los estudios ya descriptos, no toma en cuenta trabajos con pacientes en prevención primaria con arteriopatía periférica subclínica?.
El objetivo de este metaanálisis fue realizar un análisis de la bibliografía existente sobre los riesgos y beneficios del tratamiento antiagregante con aspirina en pacientes sin antecedentes cardiovasculares, con evaluación de los resultados de acuerdo con el "riesgo cardiovascular real” de las poblaciones incluidas.

\section{Material y métodos \\ Extracción de datos y evaluación de la calidad}

El metaanálisis se llevó a cabo de acuerdo con la declaración PRISMA para informar revisiones sistemáticas. Dos revisores independientes efectuaron búsquedas para identificar estudios clínicos que evaluaran el tratamiento con aspirina en comparación con placebo en paciente $\sin$ antecedentes cardiovasculares, publicados entre enero de 1988 y diciembre de 2018. Se realizaron búsquedas en las bases de datos electrónicas PubMed/Medline, EMBASE y Cochrane Clinical Trials mediante los términos "aspirina», "ácido acetilsalicílico", "prevención cardiovascular" o "prevención primaria".

La escala de Jadad se usó para evaluar la calidad del diseño de los estudios. Éstos se calificaron, en una escala de 0 a 5 , de acuerdo con la presencia de tres características metodológicas clave: aleatorización, cegamiento y pérdida en el seguimiento. Los estudios con una escala de Jadad mayores de 2 puntos se consideraron de alta calidad y se incluyeron en el análisis.

El punto final primario fue la combinación de muerte cardiovascular, infarto agudo de miocardio (IAM) y accidente cerebrovascular (ACV) isquémico. Se evaluó además como punto final de seguridad la incidencia de ACV hemorrágico y hemorragia grave, según los criterios utilizados en cada trabajo. Se clasificaron los estudios en bajo, moderado y alto riesgo, según el 
Tabla 1. Características de los estudios analizados

\begin{tabular}{|c|c|c|c|c|c|c|}
\hline & Año de publicación & Seguimiento (años) & Pacientes incluidos & Aspirina & Placebo & Riesgo cardiovascular real \\
\hline $\mathrm{BMD}^{8}$ & 1988 & 5.5 & 5,139 & 3,429 & 1,710 & Moderado \\
\hline $\mathrm{PHS}^{9}$ & 1989 & 5 & 22,071 & 11,037 & 11,034 & Bajo \\
\hline $\mathrm{HOT}^{10}$ & 1998 & 3.8 & 18,790 & 9,399 & 9,391 & Moderado \\
\hline TPT $^{11}$ & 1998 & 6.7 & 5,085 & 2,545 & 2,540 & Moderado \\
\hline PPP $^{12}$ & 2001 & 3.6 & 4,495 & 2,226 & 2,269 & Bajo \\
\hline $\mathrm{WHS}^{13}$ & 2005 & 10.1 & 39,876 & 19,934 & 19,942 & Bajo \\
\hline$J_{P A D}{ }^{14}$ & 2008 & 4.4 & 2,539 & 1,262 & 1,277 & Bajo \\
\hline POPADAD $^{15}$ & 2008 & 6.7 & 1,276 & 638 & 638 & Alto \\
\hline $\mathrm{AAA}^{16}$ & 2010 & 8.2 & 3,350 & 1,675 & 1,675 & Bajo \\
\hline JPPP17 & 2014 & 5 & 14,464 & 7,220 & 7,244 & Bajo \\
\hline ASCEN $^{4}$ & 2018 & 7.4 & 15,480 & 7,740 & 7,740 & Moderado \\
\hline ARRIVE $^{5}$ & 2018 & 5 & 12,546 & 6,270 & 6,276 & Bajo \\
\hline ASPREE $^{6}$ & 2018 & 4.7 & 19,114 & 9,525 & 9,589 & Bajo \\
\hline
\end{tabular}

MD: British Male Doctors Trial; PHS: Physicians' Health Study; HOT: Hypertension Optimal Treatment, TPT: Thrombosis Prevention Trial; PPP: Primary Prevention Project, WHS: Women's Health Study, JPAD: Japanese Primary Prevention of Atherosclerosis with Aspirin for Diabetes; POPADAD: Prevention of Progression of Arterial Disease and Diabetes; AAA: Aspirin for Asymptomatic Atherosclerosis; JPPP: Japanese Primary Prevention Project, ASCEND: A Study of Cardiovascular Events in Diabetes; ARRIVE: Aspirin to Reduce Risk of Initial Vascular Events; ASPREE: Aspirin in Reducing Events in the Elderly.

número de episodios en la rama de placebo, y se utilizaron como puntos de cortes una incidencia a 10 años menor de $10 \%$, entre 10 y $20 \%$ y mayor de $20 \%$, respectivamente.

\section{Análisis estadístico}

Las medidas del tamaño del efecto se expresaron como razón de momios (OR, odds ratios). Se evaluó la heterogeneidad a través de la prueba de Mantel-Haenszel y el método de Der Simonian-Laird y se calcularon el tau ${ }^{2}$ y el ${ }^{2}$; se rechazó la hipótesis nula de no heterogeneidad con un valor de $p \leq 0.1$. De acuerdo con la ausencia o presencia de heterogeneidad observadas en los análisis, se eligió un modelo de efectos fijos 0 aleatorios, respectivamente. Para comparar el efecto promedio entre los subgrupos se empleó una prueba Z. El nivel de significación estadística se estableció en 0.05 . Para el análisis se usó el software estadístico R.

\section{Análisis del sesgo de publicación}

Se analizó el posible sesgo de publicación con la prueba de asimetría de Egger y la representación gráfica con diagrama de embudo (funnel plot) (error estándar vs. OD).

\section{Resultados}

Mediante la búsqueda se seleccionaron los estudios aleatorizados $(n=865)$ de los 3,805 registros identificados. Luego de excluir los registros duplicados y de una primera revisión, se seleccionaron 238 estudios. De ellos, 64 artículos se evaluaron para elegibilidad y al final se extrajeron 13 estudios para el análisis cualitativo y cuantitativo (metaanálisis). Los principales motivos de exclusión fueron: a) cartas o editoriales sin datos originales; b) estudios observacionales; c) revisiones o metaanálisis; d) pacientes en prevención secundaria; e) falta de datos clínicos necesarios para el análisis; f) descripción de protocolos.

Se analizó a un total de 164,225 pacientes $(82,900$ sujetos en la rama de aspirina y 81,325 en el grupo de control). Ocho trabajos incluyeron a poblaciones con riesgo cardiovascular bajo $(n=118,455)$ y cinco con riesgo moderado/alto $(n=45,770)$. Las características de los estudios incluidos en este análisis pueden consultarse en la tabla 1. La evaluación gráfica y analítica muestra la ausencia de sesgo de publicación (Fig. 1).

En el análisis global se observó una reducción del punto final primario en el grupo de aspirina (OR, 0.90; IC 95\%, 0.85-0.94) (Fig. 2). No hubo diferencias entre los subgrupos de riesgo cardiovascular bajo y 


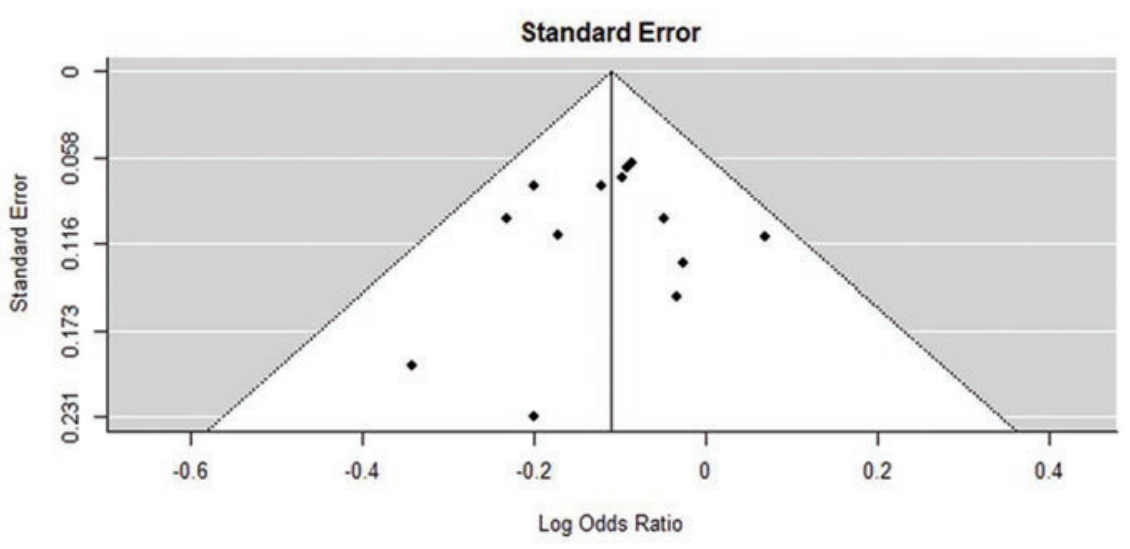

Figura 1. Diagrama de embudo: sesgo de publicación. Prueba de asimetría de Egger; $t=-0.40453, d f=11, p=0.6936$.

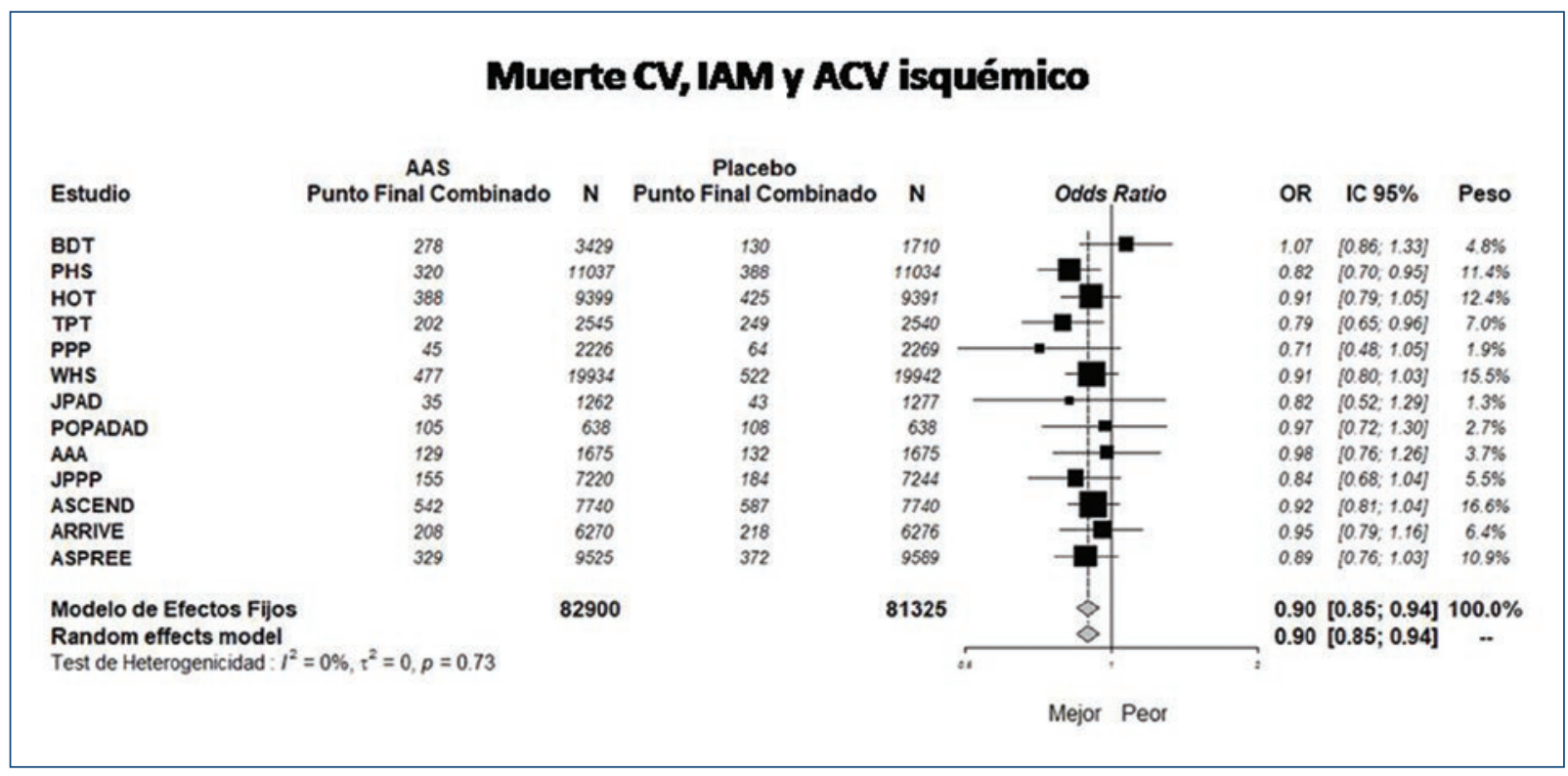

Figura 2. Gráfica de bosque. Análisis del punto final primario. Modelo de efectos fijos. CV: cardiovascular; IAM: infarto agudo de miocardio; ACV: accidente cerebrovascular.

moderado/alto (OR, 0.88; IC 95\%, 0.82-0.94 vs. OR, 0.92; IC 95\%, 0.85-0.99; $p=0.46$ ) (Fig. 3).

En el análisis individual de los episodios que conformaban el punto final combinado se observó una reducción de IAM (OR, 0.86; IC 95\%, 0.80-0.93) y ACV isquémico (OR, 0.90; IC 95\%, 0.83-0.98), sin diferencias en la mortalidad cardiovascular (OR, 0.94; IC 95\%, 0.86-1.04). El análisis de estas variables por subgrupos puede consultarse en la tabla 2.

El punto final combinado de seguridad de sangrado mayor y accidente cerebrovascular hemorrágico fue significativamente mayor en pacientes tratados con aspirina (OR, 1.45; IC 95\%, 1.32-1.60) (Fig. 4). De nueva cuenta, no se encontraron diferencias entre los subgrupos de riesgo cardiovascular bajo y moderado/alto (OR, 1.45; IC 95\%, 1.28-1.63 vs. OR, 1.48; IC 95\%, $1.21-1.80 ; p=0.84)$.

\section{Discusión}

De acuerdo con los resultados, se observó en el análisis global una menor incidencia de IAM y ACV 


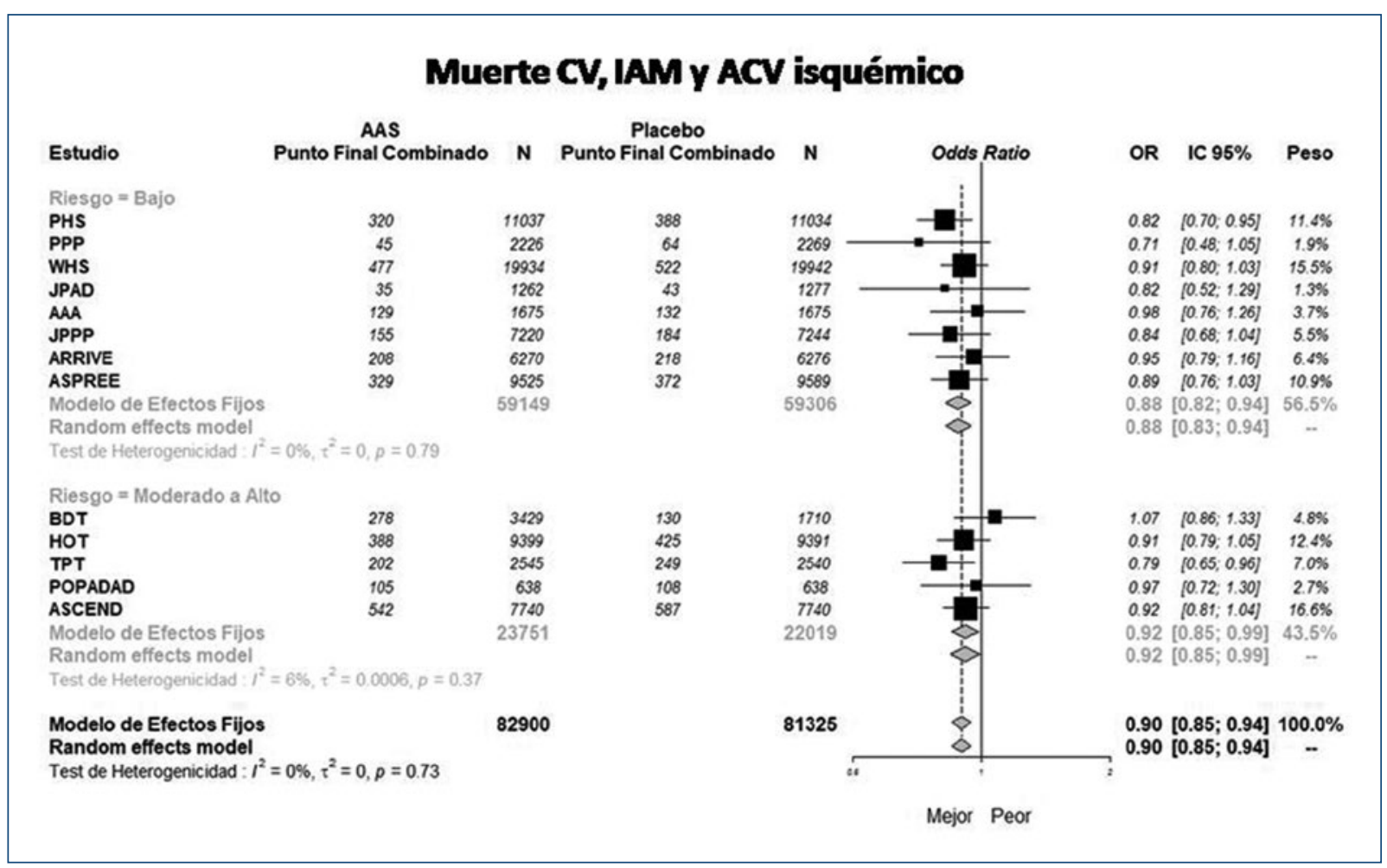

Figura 3. Gráfica de bosque. Análisis del punto final primario por subgrupos de riesgo bajo y moderado/alto. Modelo de efectos fijos. CV: cardiovascular; IAM; infarto agudo de miocardio; ACV: accidente cerebrovascular.

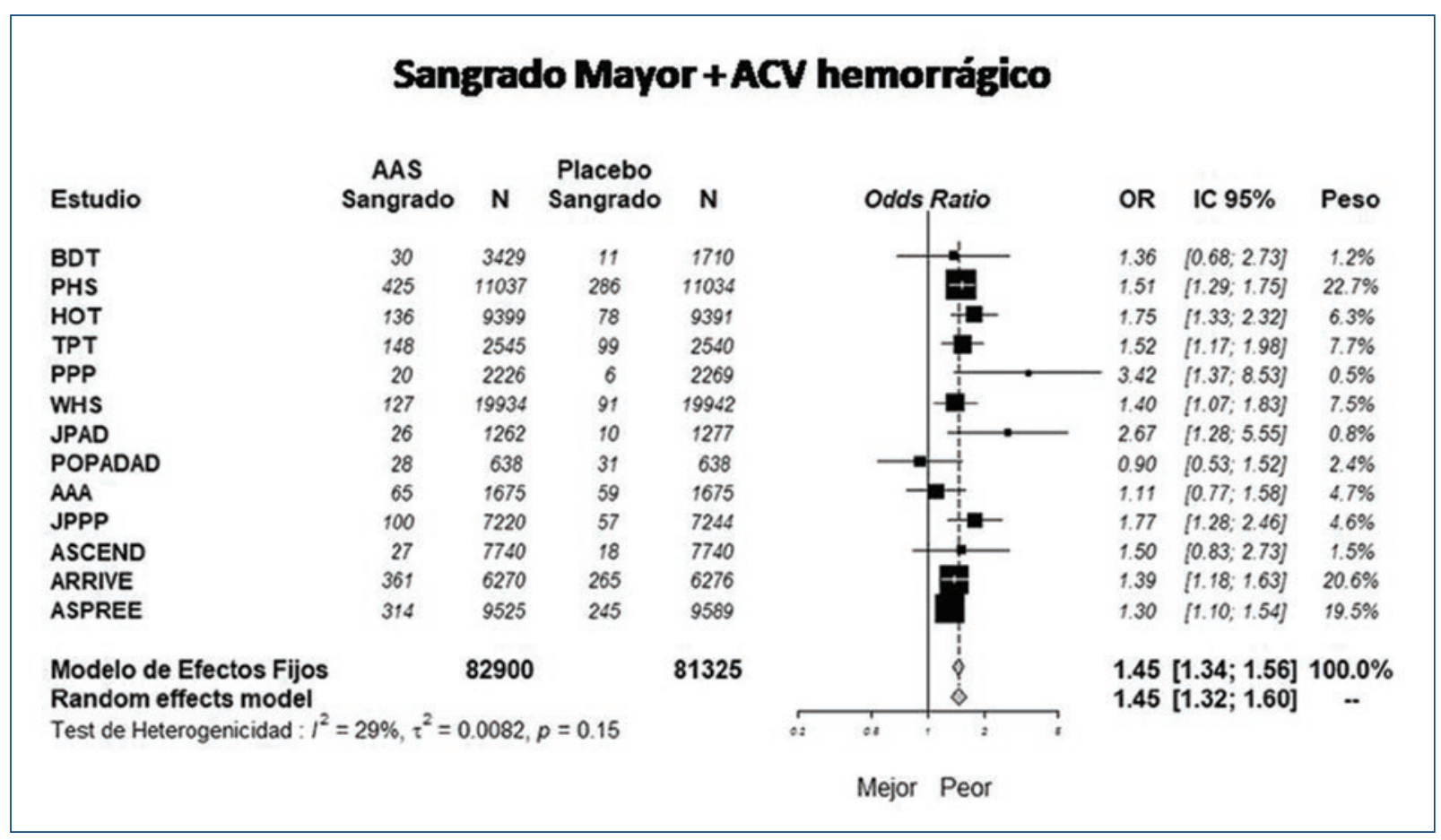

Figura 4. Gráfica de bosque. Análisis del punto final primario de seguridad (sangrado mayor + accidente cerebrovascular hemorrágico). Modelo de efectos aleatorios. ACV: accidente cerebrovascular. 
Tabla 2. Reducción de IAM, ACV y mortalidad CV por subgrupos de riesgo.

\begin{tabular}{|l|c|c|c|}
\hline & $\begin{array}{c}\text { Riesgo bajo } \\
\text { (OR; IC 95\%) }\end{array}$ & $\begin{array}{c}\text { Riesgo moderado/ } \\
\text { alto (OR; IC 95\%) }\end{array}$ & $\boldsymbol{p}$ \\
\hline IAM & $0.84(0.76-0.93)$ & $0.89(0.79-1)$ & 0.54 \\
\hline ACV & $0.91(0.82-1.02)$ & $0.89(0.78-1.01)$ & 0.76 \\
\hline Mortalidad CV & $0.89(0.77-1.03)$ & $0.99(0.87-1.11)$ & 0.27 \\
\hline
\end{tabular}

IAM : infarto agudo de miocardio; ACV: accidente cerebrovascular; CV: cardiovascular.

isquémico en pacientes tratados con aspirina, sin efectos en la mortalidad cardiovascular. Esto se acompañó de un incremento del riesgo de ACV hemorrágico y sangrado mayor.

Si bien en el análisis individual de IAM y ACV isquémico se identificó una reducción de efectos en términos relativos, la baja prevalencia en poblaciones sin antecedentes cardiovasculares da lugar a que, en términos absolutos, la disminución del riesgo tenga escasos efectos. Esto, junto con el aumento indiscutido del riesgo de sangrado, desaliente cada vez más la utilización de este fármaco en este escenario.

Según estos resultados, el número necesario a tratar para prevenir un efecto cardiovascular es de 301 y son necesarios 5,263 los pacientes para prevenir una muerte cardiovascular. Por otro lado, se necesitan sólo 157 individuos para generar un episodio de sangrado mayor. Estos datos permiten graficar de manera más clara lo descrito con anterioridad.

Muchos de los estudios que evaluaron el consumo de aspirina en prevención primaria han intentado identificar a subgrupos de alto riesgo en los que se pueda obtener una reducción de efectos más relevante en términos absolutos y lograr así el beneficio clínico neto. Para ello, distintos autores basaron sus criterios de inclusión en la presencia de factores de riesgo cardiovascular o mediante la identificación de arteriopatía periférica subclínica. Sin embargo, en ocasiones la incidencia real de episodios fue menor a la esperada. Por ejemplo, el estudio ARRIVE incluyó a hombres mayores de 55 años y mujeres mayores de 60 años con riesgo cardiovascular moderado por la presencia de factores de riesgo comunes ${ }^{5}$. Sin embargo, la incidencia de episodios calculada a 10 años de la rama de placebo fue de apenas $6.9 \%$. Otro ejemplo lo constituye el estudio ASCEND, que incluyó a pacientes diabéticos mayores de $40 a_{\text {ños }}^{4}$. Otra vez, el número de efectos fue menor al esperado y la incidencia proyectada a 10 años fue del $10.2 \%$.

Debido a estas dificultades se concedió énfasis al análisis de la evidencia disponible según el riesgo real de cada estudio. De acuerdo con el subanálisis, no existen diferencias en cuanto a la reducción de episodios entre poblaciones de bajo y moderado/alto riesgo. Más aún, se observa paradójicamente una tendencia de mayor reducción entre los trabajos de bajo riesgo, sobre todo a expensas del IAM. Por último, cabe mencionar que sólo un estudio tuvo una incidencia proyectada a 10 años mayor de 20\%. Éste mismo no mostró un beneficio claro.

Mientras que en prevención secundaria la indicación de este fármaco está consolidada, es más confusa la recomendación en la prevención primaria cuando se evalúan las principales guías internacionales. Aunque contraindican de manera unánime el uso sistemático de aspirina en la prevención primaria, aconsejan su prescripción en pacientes individualizados con alto riesgo cardiovascular y bajo riesgo de sangrado. De acuerdo con la bibliografía disponible, pondría plantearse lo siguiente: ¿quiénes son estos pacientes? Diabéticos, hipertensos, dislipémicos, adultos mayores, e incluso se ha incluido en varios trabajos a pacientes con arteriopatía periférica subclínica. Al momento, esta pregunta continua sin respuesta.

Dentro de las fortalezas de este metaanálisis figuran el gran número de pacientes analizados y la baja heterogeneidad observada en el punto final primario combinado.

Entre las limitaciones debe mencionarse la heterogeneidad en el análisis de sangrado como consecuencia de las diferentes definiciones empleadas. También se obtuvo una gran heterogeneidad en el análisis individual de IAM como consecuencia, al menos en parte, de las variaciones en la definición de IAM a lo largo del tiempo. Por último, cabe mencionar que los resultados se extrajeron de las publicaciones y no se dispuso de los datos individuales de cada paciente.

\section{Conclusión}

Como prevención primaria, el tratamiento con aspirina se vinculó con una leve disminución de IAM y $\mathrm{ACV}$ isquémico en términos absolutos, sin diferencias en la incidencia de mortalidad cardiovascular. Junto con un incremento de la incidencia de ACV hemorrágico y sangrado mayor, esto representa una ausencia de beneficio clínico neto. El riesgo cardiovascular basal de la población no modificó los resultados obtenidos. 


\section{Financiamiento}

Ninguno.

\section{Conflicto de intereses}

Los autores declaran no tener ningún conflicto de intereses.

\section{Responsabilidades éticas}

Protección de personas y animales. Los autores declaran que para esta investigación no se han realizado experimentos en seres humanos ni en animales.

Confidencialidad de los datos. Los autores declaran que en este artículo no aparecen datos de pacientes.

Derecho a la privacidad y consentimiento informado. Los autores declaran que en este artículo no aparecen datos de pacientes.

\section{Bibliografía}

1. Arnett DK, Blumenthal RS, Albert MA, Michos ED, Buroker AB, Miedema MD, et al. 2019 ACC/AHA Guideline on the primary prevention of cardiovascular disease: a report of the American College of Cardiology/American Heart Association Task Force on Clinical Practice Guidelines. J Am Coll Cardiol. 2019;pii: S0735-1097(19)33877-X.

2. Piepoli MF, Hoes AW, Agewall S, Albus C, Brotons C, Catapano AL, et al. 2016 European Guidelines on cardiovascular disease prevention in clinical practice: The Sixth Joint Task Force of the European Society of Cardiology and Other Societies on Cardiovascular Disease Prevention in Clinical Practice (constituted by representatives of 10 societies and by invited experts) Developed with the special contribution of the European Association for Cardiovascular Prevention \& Rehabilitation (EACPR). Atherosclerosis. 2016;252:207-74.

3. Valgimigli M. The remarkable story of a wonder drug, which now comes to an end in the primary prevention setting: say bye-bye to aspirin! Eur Heart J. 2019;40:618-20.
4. Bowman L, Mafham M, Wallendszus K, Stevens W, Buck G, Barton J, et al. ASCEND Study Collaborative Group. Effects of aspirin for primary prevention in persons with diabetes mellitus. $\mathrm{N}$ Engl $\mathrm{J}$ Med. 2018;379:1529-39.

5. Gaziano JM, Brotons C, Coppolecchia R, Cricelli C, Darius H, Gorelick PB, et al. Use of aspirin to reduce risk of initial vascular events in patients at moderate risk of cardiovascular disease (ARRIVE): a randomised, double-blind, placebo-controlled trial. Lancet 2018;392:1036-46.

6. McNeil JJ, Wolfe R, Woods RL, Tonkin AM, Donnan GA, Nelson MR, et al. ASPREE Investigator Group. Effect of aspirin on cardiovascular events and bleeding in the healthy elderly. N Engl J Med. 2018; 379:1509-18.

7. Mahmoud A, Gad M, Elgendy A, Elgendy I, Bavry A. Efficacy and safety of aspirin for primary prevention of cardiovascular events: a meta-analysis and trial sequential analysis of randomized controlled trials. Eur Heart J. 2019;40:607-617.

8. Peto R, Gray R, Collins R, Wheatley K, Hennekens C, Jamrozik K, et al. Randomised trial of prophylactic daily aspirin in British male doctors. $\mathrm{Br}$ Med J (Clin Res Ed). 1988;296:313-16.

9. Steering Committee of the Physicians' Health Study Research Group. Final report on the aspirin component of the ongoing Physicians' Health Study. N Engl J Med. 1989;321:129-35.

10. Hansson L, Zanchetti A, Carruthers SG, Dahlöf B, Elmfeldt D, Julius S, et al. Effects of intensive blood-pressure lowering and low-dose aspirin in patients with hypertension: principal results of the Hypertension Optimal Treatment (HOT) randomised trial. HOT Study Group. Lancet 1998;351:1755-62.

11. Thrombosis prevention trial: randomised trial of low-intensity oral anticoagulation with warfarin and low-dose aspirin in the primary prevention of ischaemic heart disease in men at increased risk. The Medical Research Council's General Practice Research Framework. Lancet 1998;351:233-41.

12. Collaborative Group of the Primary Prevention Project (PPP). Low dose aspirin and vitamin $E$ in people at cardiovascular risk: a randomised trial in general practice. Collaborative Group of the Primary Prevention Project. Lancet 2001;357:89-95.

13. Ridker PM, Cook NR, Lee IM, Gordon D, Gaziano JM, Manson JE, et al A randomized trial of low-dose aspirin in the primary prevention of cardiovascular disease in women. N Engl J Med. 2005;352:1293-1304.

14. Ogawa H, Nakayama M, Morimoto T, Uemura S, Kanauchi M, Doi N, et al. Japanese Primary Prevention of Atherosclerosis With Aspirin for Diabetes (JPAD) Trial Investigators. Low-dose aspirin for primary prevention of atherosclerotic events in patients with type 2 diabetes: a randomized controlled trial. JAMA 2008;300:2134-41.

15. Belch J, MacCuish A, Campbell I, Cobbe S, Taylor R, Prescott R, et al. The prevention of progression of arterial disease and diabetes (POPADAD) trial: factorial randomised placebo controlled trial of aspirin and antioxidants in patients with diabetes and asymptomatic peripheral arterial disease. BMJ 2008;337:a1840.

16. Fowkes FG, Price JF, Stewart MC, Butcher I, Leng GC, Pell AC, et al. Trialists AAA. Aspirin for prevention of cardiovascular events in a general population screened for a low ankle brachial index: a randomized controlled trial. JAMA 2010;303:841-48.

17. Ikeda Y, Shimada K, Teramoto T, Uchiyama S, Yamazaki T, Oikawa S, et al. Low-dose aspirin for primary prevention of cardiovascular events in Japanese patients 60 years or older with atherosclerotic risk factors: a randomized clinical trial. JAMA 2014;312: 2510-20. 